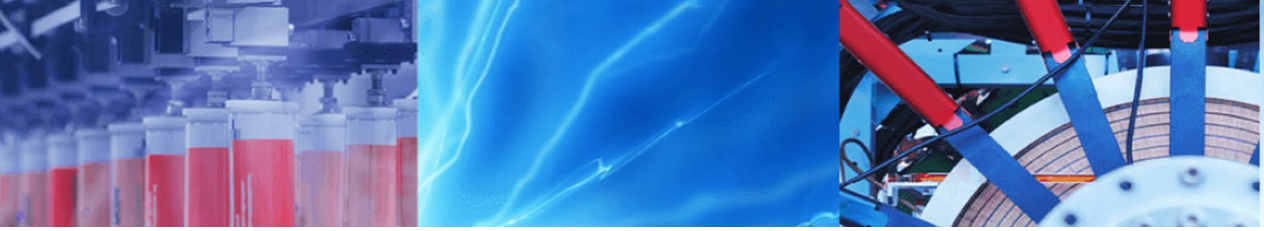

Case Study

\title{
Nonlinear models for a compressor stall phenomenon using a single pressure measurement
}

\author{
Faramarz D. Mossayebi ${ }^{1}$ D $\cdot$ Helen K. Qammar ${ }^{2}$
}

(c) Springer Nature Switzerland AG 2019

\begin{abstract}
One of the aerodynamic instabilities that severely affect the performance of a high-speed compressor is the rotating stall. In this paper, the experimental pressure measurements associated with the stall phenomenon in a compressor is analyzed. Time series analysis reveals that this process can be a nonlinear one. Furthermore, the dynamical behavior of the compressor in the stall region is shown to be chaotic. More interestingly, several deterministic polynomial models of this process, obtained from experimental pressure measurements, is reported which successfully captures the underlying global dynamics.
\end{abstract}

Keywords Time series analysis · Compressor stall model $\cdot$ NARMAX · NARX · Chaos

\section{Introduction}

The function of a compressor in an engine is to raise air pressure and temperature prior to combustion to achieve improved cycle efficiency. Since the compressor controls the pressure ratio of the engine as well as the mass flow, it exerts a dominant influence on the characteristic of gas turbine engine. Compressor have received a great deal of attention in research and development because of this influence and the fact that it has been one of the most difficult engine components to develop [1]. There are two types of compressors, axial and centrifugal. We only consider the axial compressor in this study as they are most widely used in aircraft engines and are by far the most highly developed one. A typical multistage axial flow compressor is depicted in Fig. 1 [2]. These compressors are made up of many alternating rows of blades, a rotating one followed by a stationary blade, referred to as rotors and stators, respectively.

Two aerodynamic instabilities, namely rotating stall and surge can be attributed to compressor, which severely limit its performance. Although these phenomena can be viewed as distinct or related they cannot be tolerated during normal compressor operation as they cause reduction in pressure rise, rapid heating of the blades, sever stress, etc. It must be noted that usually the onset of the local instability associated with rotating stall can trigger the more global type of system instability that leads to surge.

Although compressors have attracted a great deal of research and development no simple model that can capture the underlying dynamical instability has been reported. Most of the available models are obtained from analytical/ physical description of the process, which requires the solution of the Navier-Stokes equations by computational means. These computational fluid dynamic models are complex as consequence of solving the governing partial differential equations associated with complex geometry of system and corresponding boundary conditions.

In this study, we are mainly concerned with presenting evidence that the stall phenomenon can be characterized as a chaotic event and more importantly communicate our

This work is dedicated in memory of Dr. Hellen Killory Qammar who unfortunately passed away after a courageous battle with cancer.

$\triangle$ Faramarz D. Mossayebi, fmossayebi@ysu.edu | 'Electrical and Computer Engineering Department, Youngstown State University, Youngstown, $\mathrm{OH} 44512$, USA. ${ }^{2}$ Chemical Engineering Department, University of Akron, Akron, $\mathrm{OH} 44325$, USA.

SN Applied Sciences (2019) 1:538 | https://doi.org/10.1007/s42452-019-0548-7

Received: 13 January 2019 / Accepted: 24 April 2019 / Published online: 8 May 2019 


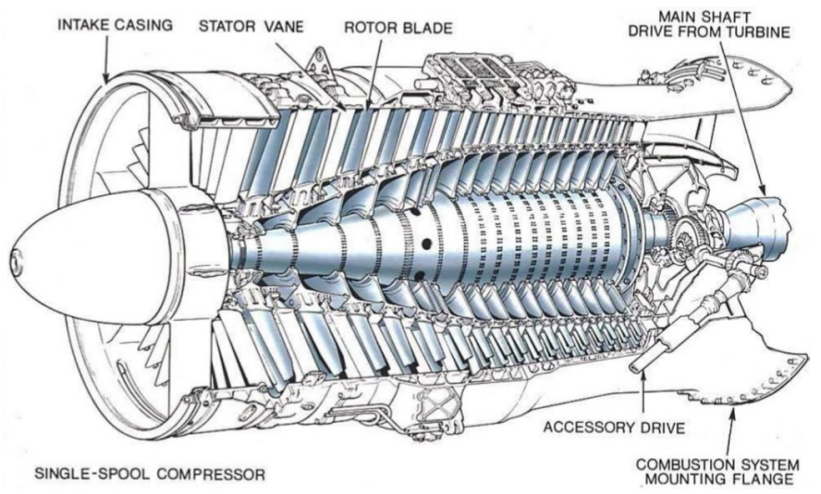

Fig. 1 A typical multistage axial flow compressor [2]

successful attempt in obtaining a simple model which captures the underlying dynamics. We believe that such characterization could lead to better understanding and to avoid (or control) this aerodynamic instability. A compressor cannot be operated in stall for more than a few seconds not only because of the safety issues involved but also to preserve the integrity of the experimental setup. This constraint limits the amount of data associated with a compressor in stall. An accurate model for a compressor in stall provides the researcher with a tool to "extend" the amount of relevant data about this phenomenon without adversely sacrificing the safety or the physical integrity of the experiment.

The paper is organized as follows. In the next section a brief account of the experimental setup is presented along with a systematic time series analysis of the pressure fluctuations of the compressor under study. Section 3 is concerned with obtaining a global model for the stall event from the pressure measurements as well as analyzing the time series obtained from the model for verification purpose. Two specific Nonlinear Auto-Regressive with eXogenous (NARX) models are presented that successfully capture the underlying dynamics. Finally, a summary of results is presented in Sect. 4.

\section{Experimental setup and time series analysis}

The compressor rig used for this study is located at NASA Glenn W-8 High-Speed Compressor Facility. The specific pressure fluctuation used for this analysis corresponds to the NASA rotor/stator pairs 35 and 37, where both stages are axial-flow, high-speed compressor stages for advanced air breathing engines. They are inlet stages designed as part of an eight-stage core compressor with an overall pressure ratio of 20:1. The compressor design is characterized by a constant mean line diameter with

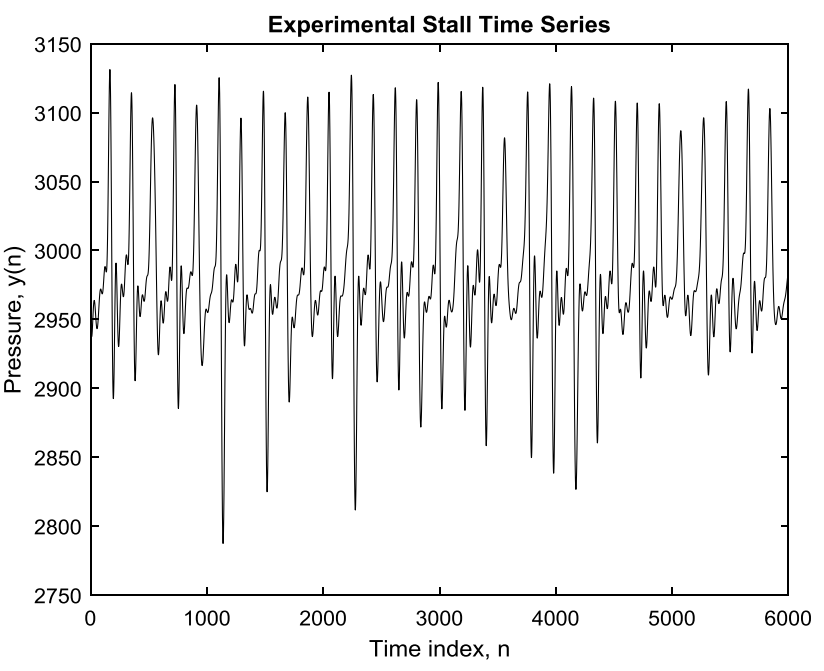

Fig. 2 Time series of the compressor in stall

an inlet hub-tip ratio of 0.7 as well as an inlet rotor-tip design speed of $455 \mathrm{~m} / \mathrm{s}$. More detailed information on the blading, geometry, and testing of this compressor can be found in references $[3,4]$.

Although 8 circumferentially placed pressure transducers within 1 chord length upstream of the rotor are used to measure pressure fluctuations, we are only concerned with one measurement from any of the sensors. These static transducers measure the pressure during transition from steady state operation into stall and surge. The pressure data acquired for this work reflects $80 \%$ speed $(140,000 \mathrm{rpm})$. The pressure measurements, which are obtained at a rate of $20 \mathrm{kHz}$, are anti-aliased and low-pass filtered prior to time series analysis. Figure 2 depicts the specific time series corresponding to the stall event measured from this experimental setup and used throughout this work.

Time series analysis techniques that have been developed by the nonlinear and chaos community seek to determine the information available in the data without predisposition to a particular type of behavior. That is, the primary objective is to obtain quantitative measure of the observed dynamics. A comprehensive review of this topic is presented by Schreiber and Kantz [5].

The first step in time series is to ascertain that the data, $p(t)$, has indeed significant nonlinearity and thus cannot be represented as a linear process containing uncorrelated noise. The surrogate test developed by Theiler et al. [6] provides a test for detecting nonlinearity in a time series. A comprehensive discussion on the proper way to obtain surrogate data is presented by Kantz and Schreiber [7], Schreiber and Schmitz [8] and Engbert [9]. Once a surrogate data is obtained then some discriminating statistic such as Kolmogorov entropy, Lyapunov 
exponents, prediction error, etc., of the data sets are used to obtain a measure of nonlinearity. We test for the null hypothesis that the data may be that of a Gaussian linear stochastic process by using both time reversal statistics and prediction error test (with a confidence level of 95\%) as advocated in Kantz and Schreiber [7]. The determination of surrogate data, like many other nonlinear time series analysis tools requires a proper embedding dimension. We will proceed with the surrogate data test results after determining this quantity of interest.

The reconstruction of the state space or the phase plane of the observed process from a single time series, $p(t)$, is the key to obtaining the desired statistics. The state space dimension, $M$, required to describe the dynamics of the observed data is referred to as the embedding dimension. The embedding dimension is the minimum number of states (or differential equations) which is required to represent the dynamics. The most practical way to obtain an $M$-dimensional vector, $\mathbf{y}(\mathbf{t})$, from the scalar time series, $p(t)$, is to simply use a sequence of lagged signals, i.e.,

$\mathbf{y}(\mathbf{t})=[p(t), p(t+T), p(t+2 T), \ldots, p(t+(M-1) T)]$.

The first minimum of the average mutual information provides a proper value for the time delay, $T$ [5]. The average mutual information associated with our data reveals that the optimum choice is $\mathrm{T}=16$ time index. The embedding dimension, $M$, can be determined by the false nearest neighbors technique [10]. The number of false neighbors is indicative of the degree to which the reconstructed attractor remains unfolded once projected to the measurement axis. The value at which the percentage of false nearest neighbors drops to zero (or its minimum for experimental data) is thus the required necessary global dimension to analyze the data. This is determined to be $M=6$ for our compressor data. With the choice of time delay, $\mathrm{T}$, and embedding $M$ at hand we can reconstruct the attractor which is known to have the same topological and geometrical properties as the original system [11]. The projection of the attractor into a 2-dimensional time delay coordinate is depicted in Fig. 3 which reveals the complicated geometrical nature the attractor associated with our compressor data. The next step in our analysis is to determine the invariants associated with the $\mathrm{M}$-dimensional reconstructed attractor.

The Lyapunov exponents are indicative of how orbits on the attractor behave under the evolution of the dynamics over a long time and provide an invariant for classifying the underlying dynamics. A positive exponent indicates that orbits will diverge while a negative exponent implies that orbits will converge. Of course, a system can be characterized as chaotic if it has at least one positive exponent, which results in sensitive dependence to initial conditions. The determination of the exponents from

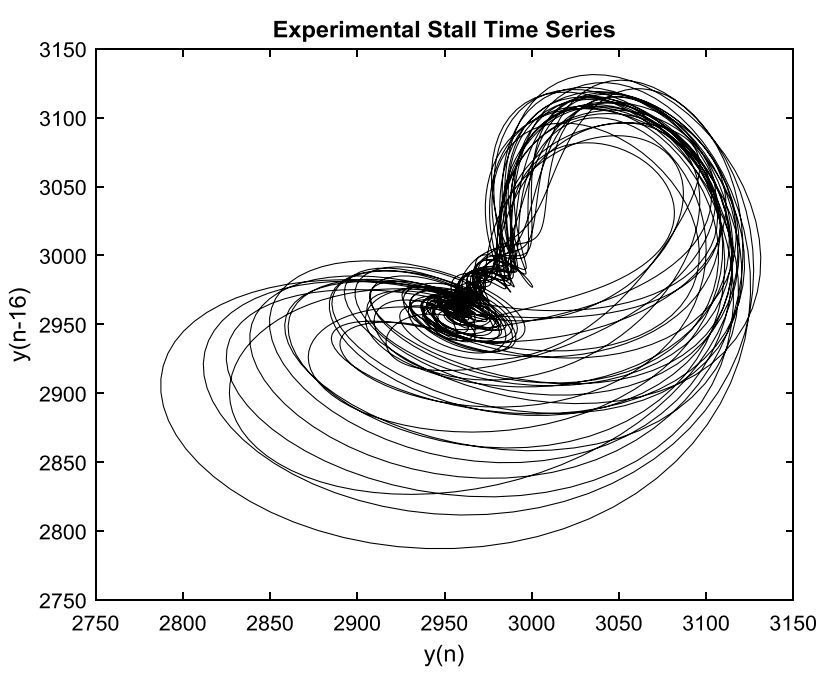

Fig. 3 The projection of the reconstructed attractor associated from the original down-sampled time series, into the delay axis

scalar measurements by using the time delay reconstruction technique is comprehensively discussed by Abarbanel [12]. The calculation of the entire spectrum of Lyapunov characteristic exponents from a time series is still a controversial topic as the quality of the data has a significant influence on the results and there is not yet a fool-proof method to distinguish the spurious exponents from the real ones [6-8]. We are actually interested in determining the largest exponents as this provides the evidence for chaos (if positive) and a tool for model validation. There are a number of algorithms for determining the largest Lyapunov exponent, we only consider the classical technique advocated by Wolf et al. [13]. We considered embedding dimensions of 6 through 8 for this task. The largest Lyapunov characteristic exponents were determined to be $0.0470,0.0463$, and 0.0458 , respectively, for the optimal choice of delay time of $T=16$, which clearly indicate a chaotic motion. It must be noted that calculations of the largest Lyapunov characteristic exponent could be influenced by the choice of time delay. We considered several other time delays; $14,15,17$, and 18 , for this calculation as well. We obtained comparative results of a positive Lyapunov exponent, using the same embedding dimensions as stated above.

The fractal dimension, $\mathrm{Dq}$, is a geometrical invariance, which characterizes the way points on the attractor are distributed. This is determined from the correlation integral, $C(r)$, which computes the fraction of pairs of $M$-dimensional points that are within a distance of $r$ from each other.

$C(r)=\frac{2}{N(N-1)} \sum_{m=1}^{N} \sum_{n=m+1}^{N} H(r-y(m)-y(n))$ 


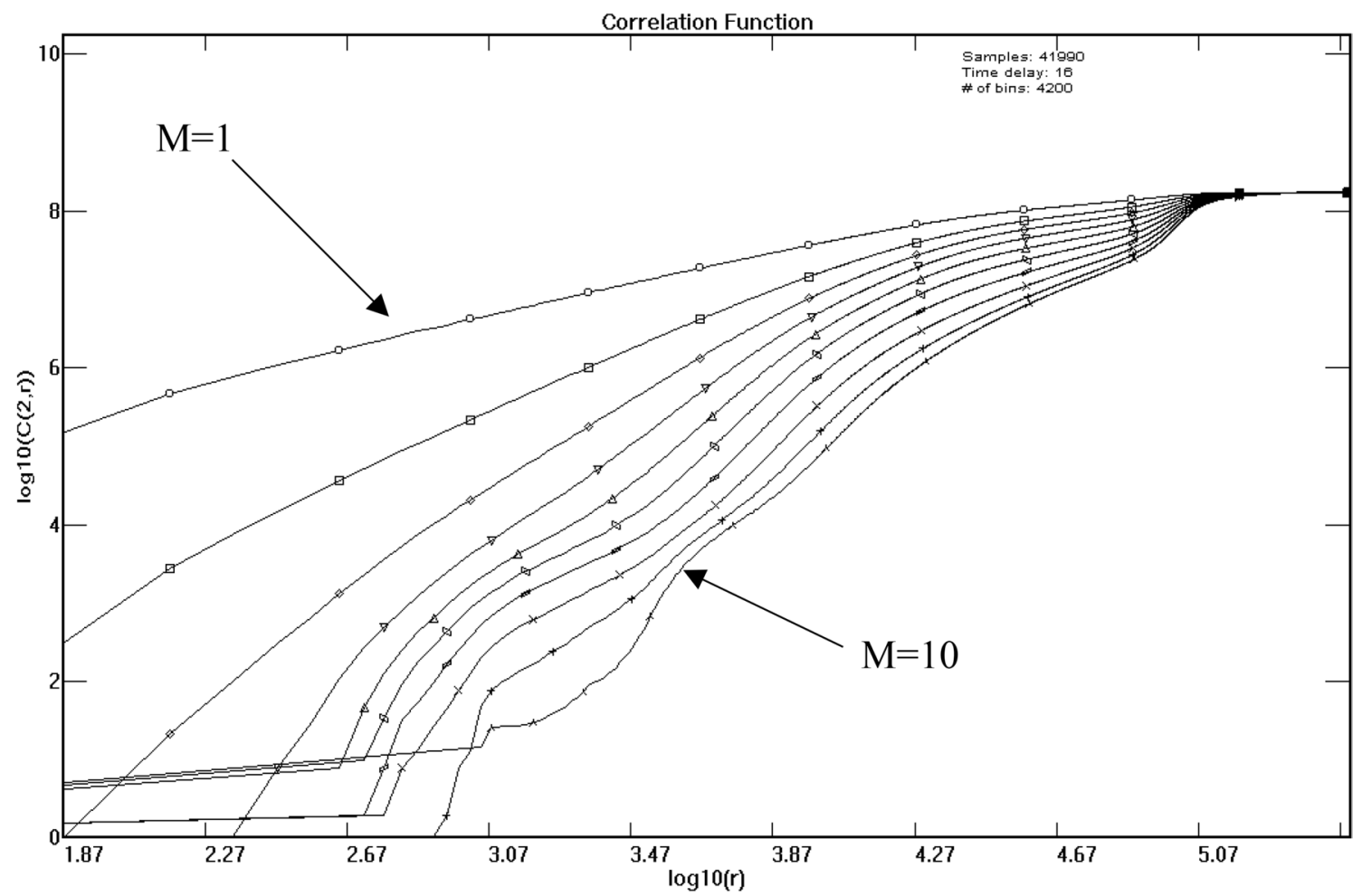

Fig. 4 The correlation integral associated with the original time series. The time delay is $T=16$ and the embedding is $M=1$ through 10

Here $\mathrm{H}$ is the Heavyside function and $\|$.$\| is the norm$ operator. Grassberger and Procaccia [14] observed that for small values of " $r$ " the correlation integral obeys a scaling law from which the fractal dimension is defined

$C(r)=r^{D_{a}}$

It must be noted that the calculation of $D_{q}$ in practice is not trivial and must be approached with great care. It turns out that the entire curve of the correlation integral in the case of real data is more prudent for analysis than just determining the slope of a portion of this curve which is indeed the dimension $D_{q}$. The determination of the correlation integral as the embedding dimension, $M$, changes provides another consistency test for embedding dimension since that curve must not be significantly changed within numerical precision (at least for "clean" data). Figure 4 shows the correlation integral associated with our pressure data for the range of $M=1$ through 10 . It can be observed that the optimum choice of embedding dimension is restricted to be between 4 and 7 which is consistent with our choice of $M=6$ as determined from the false nearest neighbors test.

With the above analysis at hand we can perform the nonlinearity tests to further confirm that the experimental data cannot be considered to be generated by stochastic process. By using the technique advocated by Schreiber and co-workers $[7,8]$, prediction error statistic, it was determined that the null hypothesis of a stationary linear Gaussian random process is rejected at the $95 \%$ level of significance (this level of confidence translates to using 40 surrogate data sets. Thus, a nonlinear event rather than a stochastic one can best describes the compressor stall phenomenon).

The time series analysis presented clearly indicates that the underlying dynamics associated with the compressor in stall is chaotic and now we turn our attention to modeling this behavior from the pressure measurement. We shall return to time series analysis in order to validate the model obtained from the pressure measurement.

\section{The modeling technique}

There has been a number of researches on the development of accurate prediction models for chaotic processes [15] but these methods result in high-order and computationally intensive models. A better approach is perhaps to treat the process as a black box and apply system identification techniques to obtain a model. In this approach first a class of models is selected and then an "appropriate" choice is made from within this class. The modeling technique that we use here indeed belongs to this category and has been shown to be quite successful in several applications [16-18]. A brief account of this modeling technique is presented in 
what follows. A comprehensive review of modeling nonlinear dynamical systems is presented by Aguirre and Letellier [19] and Billings [20].

Consider the nonlinear autoregressive moving average with exogenous inputs (NARMAX) model

$y_{k}=F^{\eta}\left[y_{k-1}, \ldots, y_{k-n_{y}}, u_{k-d}, \ldots, u_{k-n_{u}}, e_{k}, \ldots, u_{k-n_{e}}\right] \equiv F^{\eta}\left[v_{1}, \ldots, v_{s}\right]$

with $\eta$ the degree of nonlinearity of the function $F^{\eta}[],. \mathrm{n}_{\mathrm{y}^{\prime}}$ $\mathrm{n}_{\mathrm{u}}$, and $\mathrm{n}_{\mathrm{e}}$ the maximum lag for the model output, input, and noise term respectively. Since we are only interested in a deterministic model and have assumed that we have no input to the process we shall discard the moving average part of the model altogether, thus resulting in a Nonlinear Auto-Regressive with eXogenous (NARX) model, and the exogenous part will be discarded after parameter estimation. Equation (3) can be expanded by a suitable bases function, (polynomials for this study), as

$$
y_{k}=\sum_{i=1}^{s} c_{i} V_{i}+\sum_{i=1}^{s} \sum_{j=1}^{s} c_{i j} V_{i} V_{j}+\cdots+\underbrace{\sum_{i=1}^{s} \ldots \sum_{m=1}^{s} \sum_{n=1}^{s} c_{i} \ldots m n}_{\eta \text { times }} V_{i} \ldots v_{m} V_{n}
$$

with c's the parameters to be estimated and $\mathrm{s}=\mathrm{n}_{\mathrm{y}}+\mathrm{n}_{\mathrm{e}}$. Note that the process model of Eq. (4) is linear in the parameters and thus can be written in the regression form of $y(k)=\phi^{T}(k-1) \theta+\zeta(k)$ where $\phi^{T}(k-1)$ is a matrix containing the process output and noise terms up to and including time $\mathrm{k}-1$, and the elements of vector $\theta$ are the parameters associated with this matrix (the c's). The residuals, $\zeta(k)$, is defined as the difference between the measured data $y(k)$ and the one-step-ahead prediction $\emptyset^{T}(k-1) \theta$. The algorithm at hand estimates the parameters, $\theta$, by an orthogonal least square technique which minimizes the following cost function [20].

$J_{L S}(\theta)=\left|y(k)-\emptyset^{T}(k-1) \theta\right|$

This orthogonal technique remedies the structure selection problem, which is one of the major difficulties in nonlinear model identification. The parameter estimation is performed for a model of type

$y(k)=\sum_{i=1}^{n_{p}+n_{\zeta}} g_{i} \omega_{i}(k)+\zeta(k)$

where $n_{p}+n_{\zeta}$ is the number of terms in the model, $\left\{g_{i}\right\}_{i=1}^{n_{p}+n_{\zeta}}$ are parameters and polynomials $\left\{\omega_{i}(k)\right\}_{i=1}^{n_{p}+n_{\zeta}}$ are constructed to be orthogonal over the data set. The maximum mean squared prediction error, MSPE, is achieved when the model does not have any terms, i.e. $n_{p}+n_{\zeta}=0$, which is clearly equal to the mean of $\mathrm{y}^{2}(\mathrm{k})$. The MSPE will reduce by an amount equal to the mean of $g_{i}^{2} \omega_{i}^{2}(k)$ if one includes the $i$-th term, $g_{i} \omega_{i}(k)$, in the auxiliary equation. This reduction expressed as a percentage of the total MSPE gives the error reduction ratio $[21,22]$

$E R R_{i}=\frac{g_{i}^{2} \overline{\omega_{i}^{2}(k)}}{y^{2}(k)} \times 100, \quad i=1,2, \ldots, n_{p}+n_{\xi}$

Terms with large value of ERR are important and thus selected to form the model.

The application of this modeling technique to data at hand requires the selection of degree of nonlinearity, number lagged outputs and noise terms, and the number of deterministic terms in the model. Furthermore, the choice of sampling time for the time series used for this model building algorithm is crucial as discussed by Aguirre [23, 24].

Based on the rule of thumb presented in the aforementioned reference for selecting the proper sampling time we determined that our pressure data must be down sampled by a factor of three for this task. We down sampled the pressure data by a factor of three and considered two situations as far as availability of experimental data was concerned. That is, a relatively long sequence of 2000 data points and a relatively short sequence of 500 data points are considered as the input data for the modeling task. The time series analysis presented in the last section revealed that an embedding dimension of 6 is required to unfold the attractor. This information implies an upper bound on the number of lagged outputs, which is equivalent to the state space dimension of the model. An exhaustive modelling effort was then undertaken by considering the choices parameters permutations as follows: a fixed number of noise terms, 20 , for all models, number of lagged output terms from 1 through 6 , degree of nonlinearity from 2 through 6 , and number of model terms from 1 through 45, where applicable. We identified a hand full of models that can exhibit chaos but after careful examination of the associated reconstructed attractors we observed that only two models, shown in Table 1, can best represent the dynamic of stall event. The model labeled as "D6L4" was obtained by using a degree of nonlinearity of six, four lagged output, and twenty noise terms and the input sequence of 500 points, however, the model labeled as "D6L5" was obtained by using a degree of nonlinearity of six, five lagged output, twenty noise terms, and the input sequence of 2000 points. The free run time response of the models "D6L4" and "D6L5" along with the corresponding reconstructed attractors are depicted in Figs. 5 and 6, respectively. Since the original 
Table 1 The models obtained from a single pressure measurement data

\begin{tabular}{|c|c|}
\hline D6L5 & D6L4 \\
\hline$-0.51671 \mathrm{E} 1 y_{k-1}$ & $-0.12787 E 2 y_{k-1}$ \\
\hline $0.50054 \mathrm{E} 3 y_{k-2}$ & $0.34979 \mathrm{E} 2 y_{k-2}$ \\
\hline$-0.43117 E 3 y_{k-3}$ & $-0.32302 E 2 y_{k-3}$ \\
\hline $0.55706 \mathrm{E} 3 y_{k-4}$ & $0.10638 \mathrm{E} 2 y_{k-4}$ \\
\hline$-0.10033 \mathrm{E} 3 y_{k-5}$ & $0.10655 \mathrm{E}-15 y_{k-1}^{6}$ \\
\hline$-0.2797 \mathrm{E}-16 y_{k-1}^{6}$ & $-0.48919 \mathrm{E}-14 y_{k-3}^{6}$ \\
\hline $0.17466 \mathrm{E}-11 y_{k-2} y_{k-1}^{4}$ & $0.29074 \mathrm{E}-13 y_{k-4}^{2} y_{k-3} y_{k-2}^{3}$ \\
\hline$-0.16182 \mathrm{E}-9 y_{k-5}^{4}$ & $0.1205 E-13 y_{k-4} y_{k-3}^{4} y_{k-1}$ \\
\hline $0.3701 \mathrm{E}-7 y_{k-3} y_{k-1}^{3}$ & $0.97008 \mathrm{E}-12 y_{k-2}^{5}$ \\
\hline $0.22724 \mathrm{E}-5 y_{k-5}^{2} y_{k-4}$ & $-0.73338 \mathrm{E}-14 y_{k-4}^{4} y_{k-1}^{2}$ \\
\hline $0.13559 y_{k-4} y_{k-3}$ & $0.26176 \mathrm{E}-13 y_{k-4} y_{k-3}^{4} y_{k-2}$ \\
\hline $0.19997 \mathrm{E}-4 y_{k-5} y_{k-1}^{2}$ & $0.292 \mathrm{E}-14 y_{k-4}^{2} y_{k-3}^{3} y_{k-1}$ \\
\hline$-0.16106 \mathrm{E}-1 y_{k-5} y_{k-2}$ & $-0.29512 \mathrm{E}-14 y_{k-4} y_{k-3} y_{k-2} y_{k-1}^{3}$ \\
\hline$-0.35265 \mathrm{E} 6$ & $0.30359 \mathrm{E}-13 y_{k-4}^{3} y_{k-3} y_{k-2} y_{k-1}$ \\
\hline$-0.90257 \mathrm{E}-8 y_{k-4} y_{k-1}^{3}$ & $0.7986 \mathrm{E}-14 y_{k-4} y_{k-3}^{5}$ \\
\hline $0.34061 \mathrm{E}-15 y_{k-5} y_{k-1}^{5}$ & $-0.39634 \mathrm{E}-13 y_{k-4}^{3} y_{k-2}^{3}$ \\
\hline $0.26426 \mathrm{E}-4 y_{k-2}^{2} y_{k-1}$ & $0.12862 \mathrm{E}-13 y_{k-4} y_{k-3}^{3} y_{k-1}^{2}$ \\
\hline $0.20295 \mathrm{E}-1 y_{k-5} y_{k-3}$ & $-0.78849 \mathrm{E}-13 y_{k-4}^{2} y_{k-3}^{2} y_{k-2} y_{k-1}$ \\
\hline $0.16767 \mathrm{E}-11 y_{k-4} y_{k-1}^{4}$ & $0.63784 \mathrm{E}-6 y_{k-2} y_{k-1}^{2}$ \\
\hline$-0.66765 \mathrm{E}-11 y_{k-3} y_{k-1}^{4}$ & $-0.75456 \mathrm{E}-14 y_{k-3}^{4} y_{k-2}^{2}$ \\
\hline$-0.26673 \mathrm{E}-4 y_{k-3} y_{k-1}^{2}$ & $0.29942 \mathrm{E}-13 y_{k-4}^{3} y_{k-2}^{2} y_{k-1}$ \\
\hline$-0.16186 \mathrm{E}-11 y_{k-5} y_{k-1}^{4}$ & $-0.71187 \mathrm{E}-12 y_{k-3}^{2} y_{k-2} y_{k-1}^{2}$ \\
\hline$-0.11393 \mathrm{E}-8 y_{k-1}^{4}$ & $0.29878 \mathrm{E}-6 y_{k-1}^{3}$ \\
\hline$-0.70602 \mathrm{E}-1 y_{k-4}^{2}$ & $-0.31904 \mathrm{E}-11 y_{k-4} y_{k-2}^{2} y_{k-1}^{2}$ \\
\hline$-0.69253 \mathrm{E}-9 y_{k-5}^{3} y_{k-1}$ & $0.59349 \mathrm{E}-14 y_{k-4}^{4} y_{k-2}^{2}$ \\
\hline $0.58126 \mathrm{E}-6 y_{k-5}^{2} y_{k-2}$ & $0.20998 \mathrm{E}-15 y_{k-2}^{4} y_{k-1}^{2}$ \\
\hline$-0.33443 y_{k-4} y_{k-2}$ & $-0.46635 \mathrm{E}-15 y_{k-4}^{6}$ \\
\hline$-0.42485 \mathrm{E}-4 y_{k-2} y_{k-1}^{2}$ & $0.31594 \mathrm{E}-11 y_{k-4} y_{k-3} y_{k-1}^{3}$ \\
\hline $0.59061 \mathrm{E}-4 y_{k-4} y_{k-2}^{2}$ & $-0.76477 \mathrm{E}-9 y_{k-4} y_{k-1}^{3}$ \\
\hline$-0.31292 \mathrm{E}-5 y_{k-3}^{2} y_{k-1}$ & \\
\hline $0.14028 \mathrm{E}-5 y_{k-5}^{2} y_{k-1}$ & \\
\hline $0.10801 \mathrm{E}-9 y_{k-5} y_{k-4} y_{k-3} y_{k-2}$ & \\
\hline$-0.13809 \mathrm{E}-7 y_{k-2}^{3} y_{k-1}$ & \\
\hline $0.1784 \mathrm{E}-11 y_{k-2}^{3} y_{k-1}^{2}$ & \\
\hline $0.41571 \mathrm{E}-9 y_{k-5}^{2} y_{k-4} y_{k-3}$ & \\
\hline$-0.13505 \mathrm{E}-11 y_{k-5}^{2} y_{k-3} y_{k-2} y_{k-1}^{2}$ & \\
\hline $0.31505 \mathrm{E}-12 y_{k-5}^{2} y_{k-3}^{2} y_{k-1}$ & \\
\hline$-0.53104 \mathrm{E}-12 y_{k-5} y_{k-3}^{4}$ & \\
\hline$-0.58495 \mathrm{E}-9 y_{k-3}^{4}$ & \\
\hline $0.12386 \mathrm{E}-15 y_{k-5} y_{k-3}^{3} y_{k-2} y_{k-1}$ & \\
\hline
\end{tabular}

measurement was down-sampled by a factor of three for modeling purpose, we must use a delay time of $T=16 / 3 \cong 5$ for reconstruction of the attractor. Thus, although for Fig. 3 a time delay of $\mathrm{T}=16$ is used, for
Figs. 5 and 6 a time delay of $T=5$ is used because of the down sampling. Comparisons of Fig. 3 with Figs. 5 and 6 reveals that the reconstructed attractors associated with the measurement and the models closely resemble each other. This visual inspection can only be used to exclude models in validation process. Unfortunately, most conventional validation approaches are not particularly attractive when the models are chaotic, thus alternative invariants must be considered to quantify the quality and adequacy of the estimated models. We consider metrics for the time series analysis just presented as tools for validating the estimated models.

In order to repeat our time series analysis, we generate $\mathbf{2 0 0 0}$ data points from the discrete time models of the compressor depicted in Table 1 by using initial conditions from the original data. The mutual information and False nearest neighbors associated with both models are identical to that of the original data considering the effect of down sampling performed for model purposes. The largest Lyapunov characteristic exponents of the models "D6L4" and "D6L5" using Wolf's algorithm are 0.0339 and 0.0302 , respectively, using an embedding dimension of 7 and time delay of $\mathrm{T}=5$, which are close to that obtained from down-sampled experimental data of 0.0463. Clearly, the model "D6L4" has a "better" maximum exponent (closer to that of experimental data) and has a reconstructed attractor that more closely resemble the experimental one compared to that of "D6L5" model. For the sake of completeness, the correlation integrals associated with the two models are also determined as depicted in Fig. 7. Considering the scaling region of the correlation integral curves of Figs. 4 and 7 also reveals that there is a close similarity between those of the original and the models. In another words the models and the experimental data have similar correlation dimensions.

The analysis just presented on these two sets of data which are generated by free run simulation of the models indicate that they capture the underlying dynamics associated with the experimental data and thus these models can be considered to be valid models for stall event in the particular compressor at hand.

\section{Discussion}

In this paper, we have analyzed the pressure fluctuations of a high-speed compressor which was experimentally forced to exhibit the rotating stall phenomenon. Our time series analysis shows that a nonlinear process can best describe this aerodynamic instability. The surrogate data 
Fig. 5 The free run time response of the model D6L4 of Table 1 and the corresponding reconstructed attractor. The model only requires four initial conditions which, are picked from the original time series
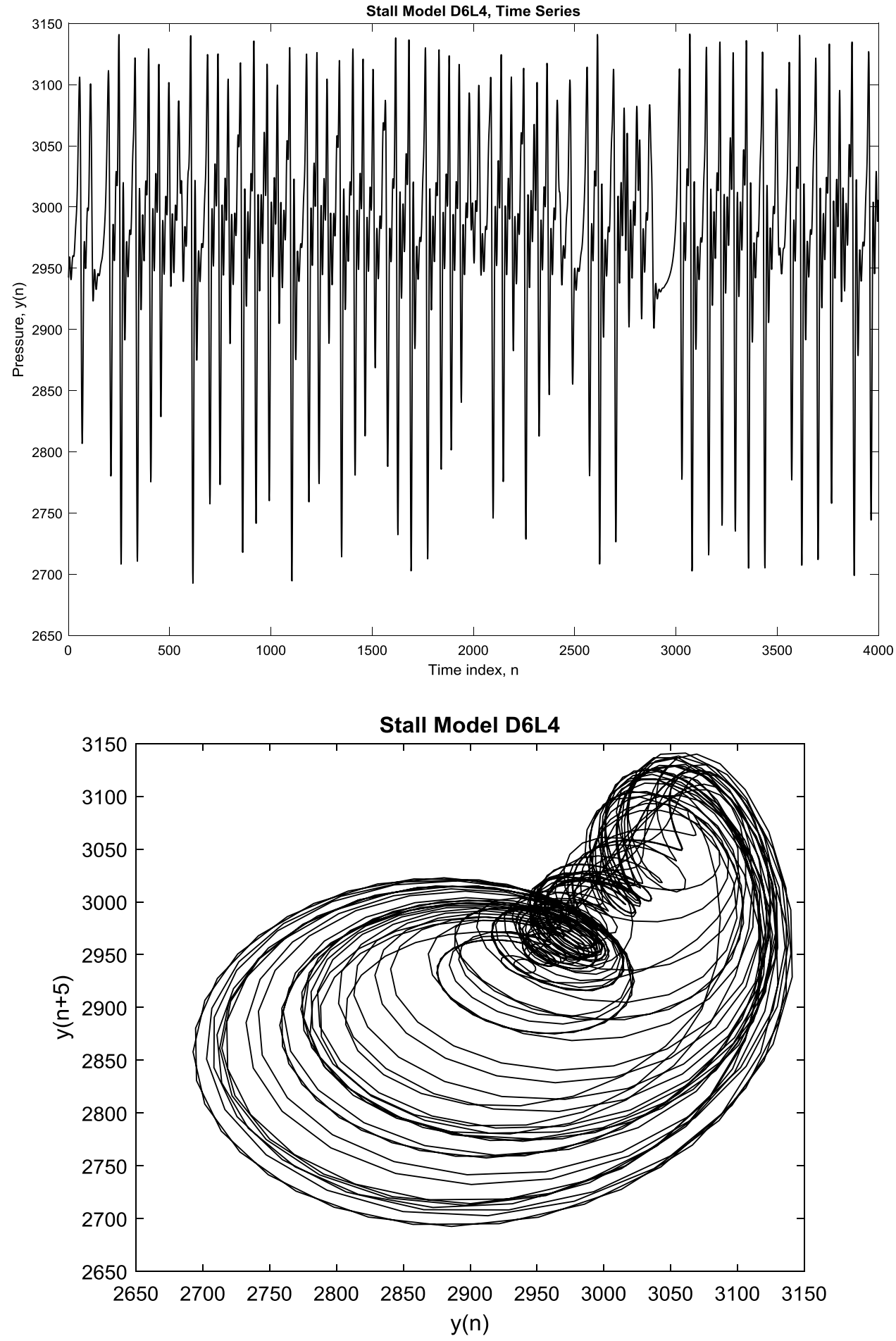

analysis rules out the hypothesis that the process can be described as a linear stochastic process and the existence of at least one positive Lyapunov exponent is indicative of the chaotic nature of this phenomenon. Moreover, global models that can successfully capture the underlying geometrical nature of the reconstructed attractor associated with stall has been reported. This is verified by the observation that the signals generated by the models (free run simulations) and the experimental measurements have the same geometrical properties. Interestingly, one of the models reported, labeled as D6L5 in Table 1, was obtained by using a small subset of the experimental data. That is, 
Fig. 6 The free run time response of the model D6L5 of Table 1 and the corresponding reconstructed attractor. The model only requires five initial conditions which, are picked from the original time series
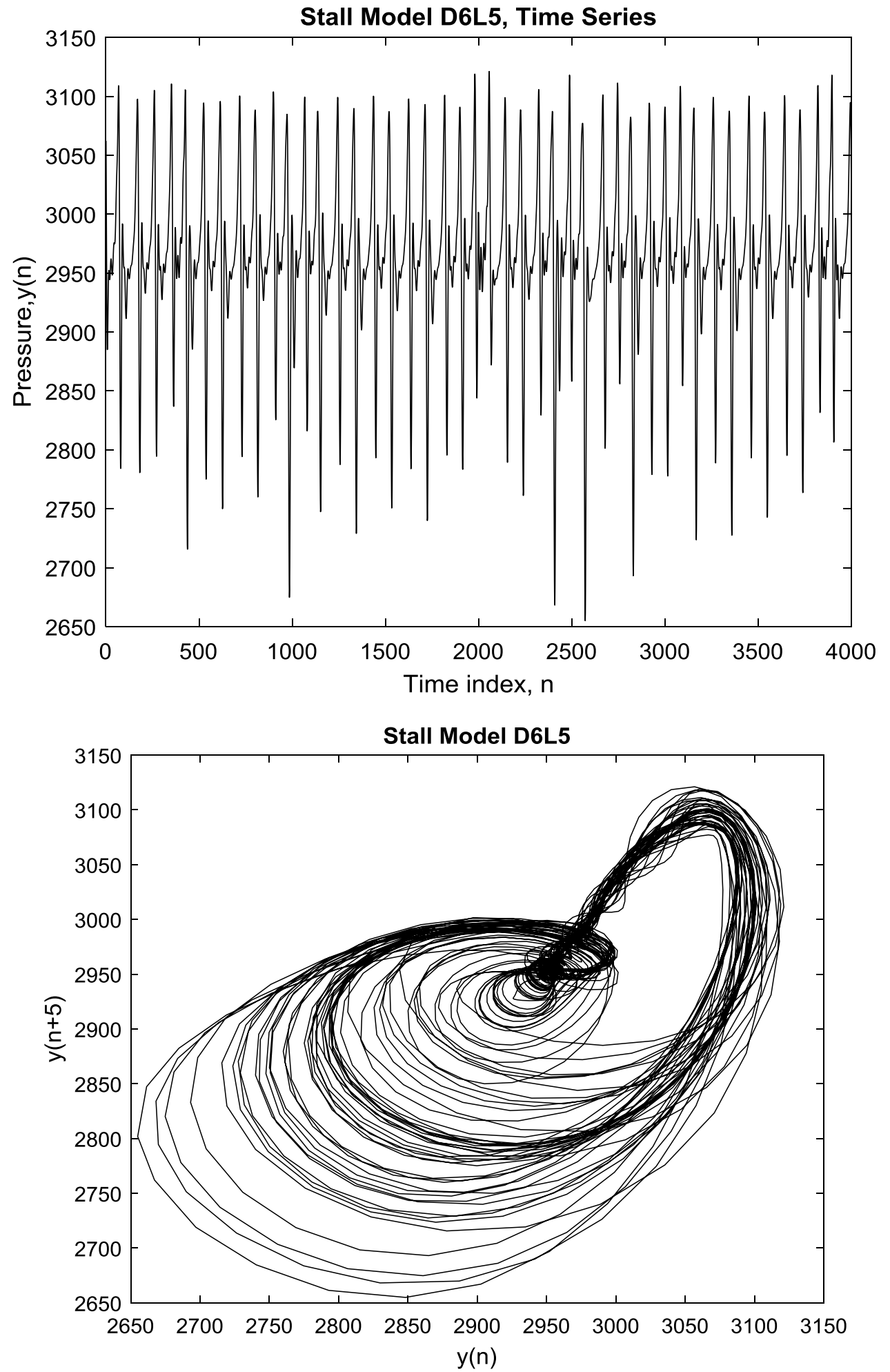

the amount of data used to generate this model corresponds to roughly $0.1 \mathrm{~s}$ of compressor operated in stall. This modeling result enables the compressor designers to obtain an extended data associated with the stall phenomenon for further analysis without adversely affecting the compressor rig. 
Fig. 7 The correlation integral obtained from the free run the time series of the models of Table 1 with a time delay of $\mathrm{T}=5$ and the embedding of $M=1$ through 10

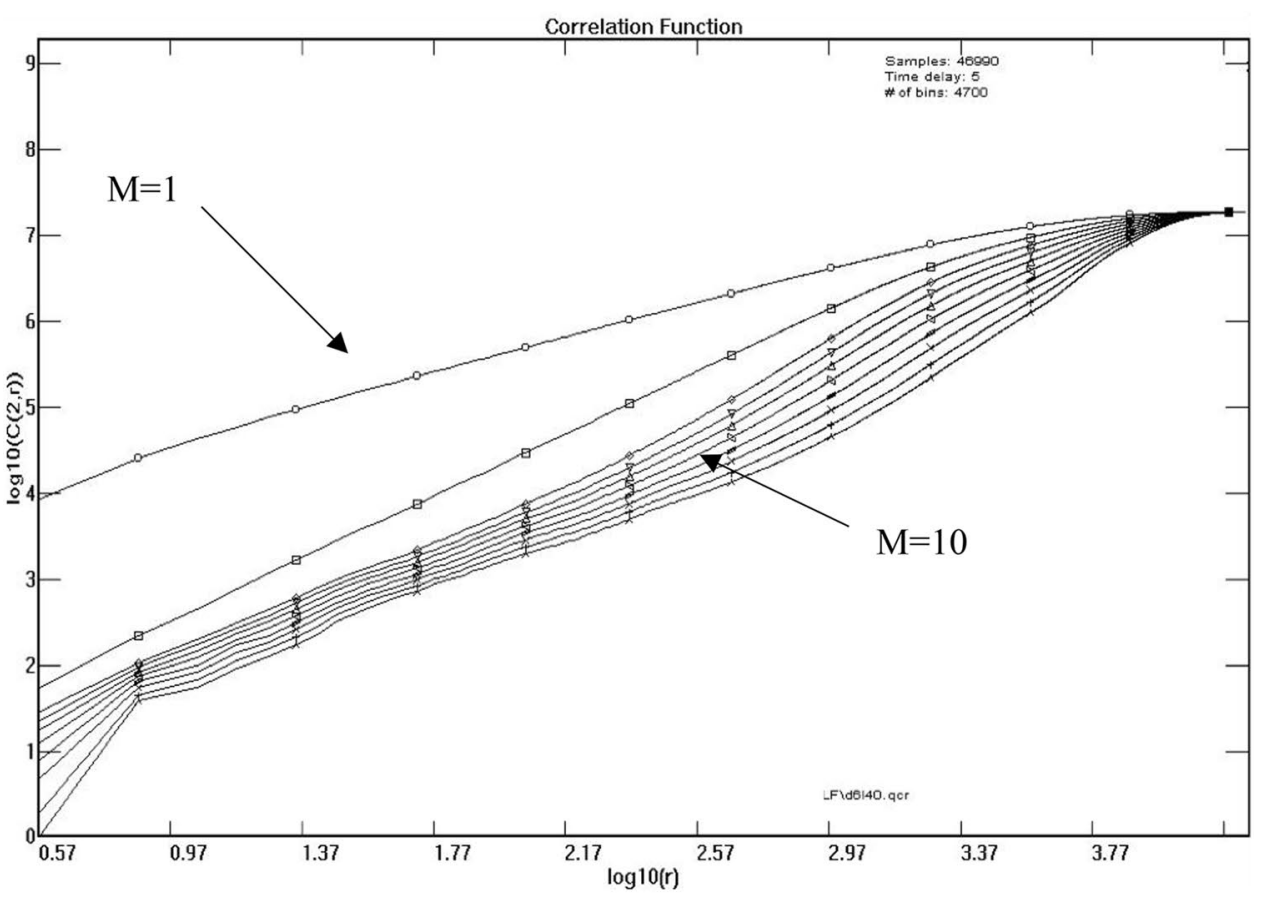

(a) Model D6L4

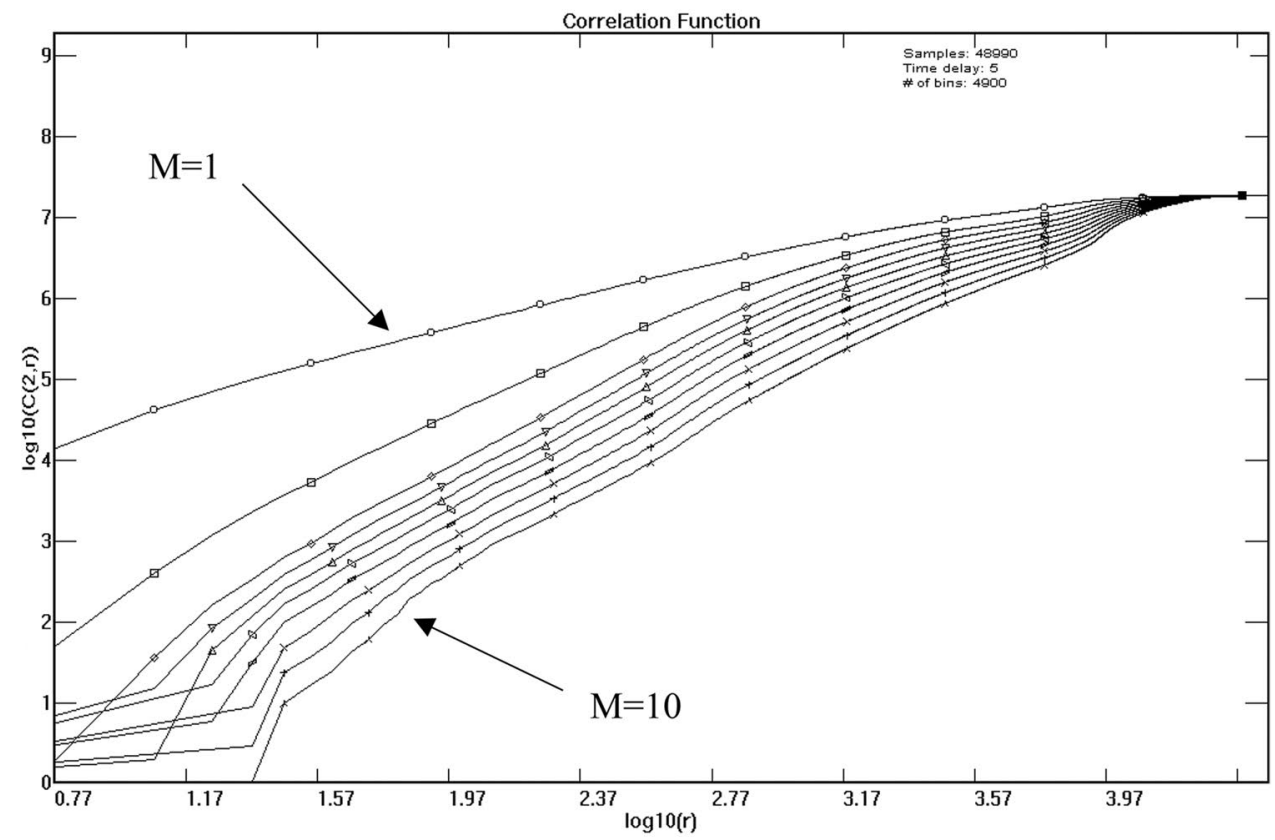

(b) Model D6L5
Acknowledgements We are greatly indebted to Professor Luis A. Aguirre for providing the MATLAB based software for NARMA modeling and Professor Eduardo M. A. M. Mendez for fruitful discussions. The Compressor data was provided by Dr. Michelle Bright while she was employed by NASA. This research has been partially supported by NSF Grant CTS-9502327.

\section{Compliance with ethical standards}

Conflict of interest On behalf of all authors, the corresponding author states that there is no conflict of interest. 


\section{References}

1. Kerrebrock JL (1987) Aircraft engines and gas turbines. MIT Press, Cambridge

2. Denton JD, Dawes WN (1990) Computational fluid dynamics for turbomachinery. Proc Inst Mech Eng Part C J Mech Eng Sci 213:107-124

3. Reed L, Moore RD (1978) Design and overall performance of four highly loaded, high-speed inlet stages for an advanced highpressure ratio core compressor. NASA Technical Paper 1337

4. Moore RD, Reid L (1980) Performance of single-stage axial-flow transonic compressor with rotor and stator aspect ratios of 1.19 and 1.26 , respectively, and with design pressure ratio of 2.05 . NASA Technical Paper 1659

5. Schreiber T, Kantz H (2005) Nonlinear time series analysis, 2nd edn. Cambridge University Press, Cambridge

6. Theiler J, Galdrikian B, Eubank S, Farmer JD (1991) Using surrogate data to detect nonlinearity in time series. In: Proceedings of nonlinear modeling and forecasting, Santa Fe Institute Studies in the Science of Complexity, vol XII. Addison-Wesley

7. Kantz H, Schrieber T (1997) Nonlinear time series analysis. Cambridge University Press, Cambridge

8. Schreiber T, Schmitz A (1996) Surrogate time series. Phys Rev Lett 77(4):635

9. Engbert R (2002) Testing for nonlinearity: the role of surrogate data. Chaos Solitons Fractals 13(1):79-84

10. Abarbanel HDI, Kennel MB (1993) Local false neighbors and dynamical dimensions from observed chaotic data. Phys Rev $E$ 47:3057

11. Sauer T, York JA, Casdagli MJ (1991) Embedology. J Stat Phys 65:579-616

12. Abarbanel HDI (1995) Analysis of observed chaotic data. Springer, New York
13. Wolf A, Swift JB, Swinney HL, Vastano JA (1985) Determining Lyapunov exponents from a time series. Physica D 16:285

14. Grassberger P, Procaccia I (1983) Measuring the strangeness of strange attractor. Physica D 9(1-2):189-208

15. Abarbanel HDI (2013) Predicting the future: completing models of observed complex systems. Springer, Berlin

16. Aguirre LA, Billings SA (1995) Closed-loop suppression of chaos in nonlinear driven oscillators. J Nonlinear Sci 5:189-206

17. Aguirre LA, Billings SA (1994) Validating identified nonlinear models with chaotic dynamics. Int J Bifurc Chaos 4(1):109-125

18. Ahmad Y, Lo M (2014) Nonlinear time series models and model selection, chapter 6. In: Ma J, Yohar M (eds) Recent advances in estimating nonlinear models. Springer, Berlin

19. Aguirre LA, Letellier C (2009) Modeling nonlinear dynamics and chaos: a review. Math Probl Eng. https://doi. org $/ 10.1155 / 2009 / 238960$

20. Billings SA (2013) Nonlinear system identification: NARMAX methods in the time, frequency, and spatio-temporal domains. Wiley, Hoboken

21. Korenberg IJ, Billings SA, Liu YP, Mcilroy PJ (1988) Orthogonal parameter estimation algorithm for nonlinear stochastic systems. Int J Control 48(1):193-210

22. Billings SA, Chen S, Korenberg IJ (1989) Identification of nonlinear systems using a forward-regression orthogonal estimator. Int J Control 49(6):2157-2189

23. Aguirre LA (1994) Some remarks on structure selection for nonlinear models. Int J Bifurc Chaos 4(6):1707-1714

24. Aguirre LA (1997) On the structure of nonlinear polynomial models: higher order correlation functions, spectra, and term clusters. IEEE Trans Circuits Syst I Fundam Theory Appl 44(5):450-453

Publisher's Note Springer Nature remains neutral with regard to jurisdictional claims in published maps and institutional affiliations. 